\title{
Interaction between Sleep EEG and ECG Signals during and after Obstructive Sleep Apnea Events with or without Arousals
}

\author{
AH Khandoker, CK Karmakar, M Palaniswami \\ The University of Melbourne, Victoria, Australia
}

\begin{abstract}
This is a preliminary attempt to directly investigate the interactions of sleep EEG and ECG signals during normal, OSA breathing event and events following its termination with or without arousal in non-REM(NREM) and REM sleep stages. ECG and EEG signals were collected from 10 patients with OSA and 5 healthy subjects. Coherence between two signals (Coherence ${ }_{E C G-}$ $E E G)$ over different frequency bands(range: $0 \sim 40 \mathrm{~Hz}$ ) were calculated for normal breathing events, OSA events and events following OSA terminations (with/without arousals) in NREM as well as REM sleep. In normal breathing events, overall Coherence ${ }_{E C G-E E G}$ in REM sleep is higher than that in NREM sleep. Significant $(p<0.01)$ differences of Coherence ${ }_{E C G-E E G}$ between OSA events with and without arousals were found in NREM sleep over 0.5-25 Hz bands but in REM sleep over 3.0-12 Hz. This research could be useful in understanding cardiac dysfunction in sleep apnoea patient.
\end{abstract}

\section{Introduction}

Obstructive sleep apnea (OSA) is characterized by recurrent episodes of upper airway collapse and obstruction during sleep. These episodes of obstruction are associated with recurrent oxyhemoglobin desaturation and arousals from sleep. Recently, effects of OSA on the interaction between cardiac autonomic control and EEG have been studied [1-2]. Jurysta et al. [2] reported that in patients with severe obstructive sleep apnea syndrome (OSAS), coherence between the autonomic cardiac fluctuations and delta sleep EEG was decreased due to a loss of heart rate variability (HRV) during shifts in sleep stages (non REM and REM).

Arousal from sleep is an important mechanism for reestablishing upper airway patency in OSA. When it occurs during sleep, brainstem centers may be activated firstly, driving tonic neuronal activity and inducing the appearance of a coupled rhythm expressed in tachycardia and synchronized EEG arousal. Around 30\% of apneas are not terminated by visible cortical arousals [3-4] based on sudden shift in EEG frequency. When arousals are not seen at event termination, it is presumed that they exist but we failed to detect them.

During sleep, brain (i.e. central nervous system: brain and spinal cord) mediates with general homeo-dynamics (controlled by the peripheral nerves of the autonomic system, i.e. sympathetic + para-sympathetic). The brain electrical activity accompanying cardio-afferent input has been studied to elucidate the processing of signals of cardiac origin [5-6] for cardiac arrhythmias. Abnormality in nerve conduction in efferent and afferent pathways innervating the heart thus could be thought as the early sign of cardiac disease in OSAS patients because abnormal afferent feedback signals may drive proarrhythmic autonomic effects on the heart, most likely interacting at the level of the brainstem.

Given above background information, it could be hypothesized that there may be differences in the effects of OSA events on the interaction between sleep EEG and ECG signals in sleep stages (non-REM and REM). However, there may be differences between events and sleep stages in arousability or in the visibility of arousals. In this study, we, therefore, characterize the coherence of EEG and ECG signals during normal, OSA breathing event and following its termination with or without arousal in non- REM and REM sleep stages.

\section{Methods}

\subsection{Subjects and sleep studies}

The polysomnograms of 10 OSAS patients (OSAS+), (mean \pm SD) age $54 \pm 9$ yrs, body mass index (BMI) $30 \pm 2$ $\mathrm{kg} / \mathrm{m} 2$, and five healthy subjects (OSAS-), age $51 \pm 8 \mathrm{yrs,}$ BMI $28.5 \pm 2 \mathrm{~kg} / \mathrm{m} 2$ were analysed. All subjects were free of any cardiac history. Diagnosis was based on clinical symptoms and polysomnographic (PSG) outcomes. PSG study included electroencephalogram (channel C3- 
A2/C4-A1; sampling frequency $=256 \mathrm{~Hz}$ ), left and right electrooclugram, leg movements, body positions, thoracic and abdominal wall expansion (by respiratory inductive plethysmography), oronasal airflow (by Nasal pressure, Pnasal), arterial oxygen saturation $\mathrm{SaO} 2$ (by pulse oximetry), submental electromyography (EMG) and ECG (sampling frequency $=256 \mathrm{~Hz}$ with a resolution of 16bits/sample). Respiratory events were scored using criteria proposed by the AASM[7-8]. In OSAS patients, apnea was defined as the complete cessation of breathing for $>10 \mathrm{sec}$ associated with a larger decrease than $3 \%$ in arterial $\mathrm{O}_{2}$ saturation or an arousal. Apneas are all obstructive. In this study, apnea hypopnea index (AHI) $>10$ was chosen as the threshold to identify the presence of OSAS + and AHI $<5$ for OSAS-. Five classical sleep stages are defined from an EEG recording. Each period of $30 \mathrm{~s}$ is visually scored as stage $1,2,3,4$, or REM (retinal eye movement) sleep. Awake states are also scored, in agreement with the criteria cited above. Stages 1-4 were combined to define non-REM (NREM) sleep.

\subsection{Coherence analysis}

This study highlights the dynamic interaction of heart and brain in sleep disordered breathing events with or without arousals. ECG and EEG signals during NREM and REM sleep were examined for location of the pre-scored apnea events. From NREM sleep, total 54/98 clips (20-second simultaneous sample of ECG and EEG signals) during OSA and 54/98 clips following termination of OSA events with/without arousals were extracted. Similarly from REM sleep, total 24/59 clips (20-second simultaneous sample of ECG and EEG signals) during OSA and $24 / 59$ clips following termination of OSA events with/without arousals were extracted. For comparison, 81 clips during normal breathing events in NREM and 108 clips in REM sleep were selected from OSAS- subjects. An arousal was scored when an abrupt shift to a faster EEG frequency (including theta, alpha and /or greater frequencies but no spindles) occurred and lasted 3-15 sec which was based on ASDA definition [9]. Coherence between EEG and ECG signals (Coherence $_{E C G-E E G}$ ) for normal, OSA and termination events during NREM and REM sleep over the frequency band $(0-128 \mathrm{~Hz})$ was obtained by using the following equation for analyzing the relationship between ECG and EEG signals in the frequency domain. The magnitude of coherence was computed on each 5-second data window and averaged over 20-second epochs. The coherence spectra band was calculated for 20-second ECG and EEG samples during OSA and following its termination with/without arousal. Cross power spectral density (distribution of power per unit frequency) of the ECG and EEG signals was calculated using the Welch's averaged modified periodogram method of spectral estimation. The magnitude of coherence is a function of frequency with values between 0 and 1 that indicates how well two signals correspond to each other at each frequency. Coherence $=1$ means the two signals are related and 0 indicates the independence of the two signals. The coherence was grouped into 5 conventional bands[1]: delta $[0.5-3.0 \mathrm{~Hz}]$, theta $[3.0-8.0 \mathrm{~Hz}]$, alpha $[8.0-12.0$ $\mathrm{Hz}]$, sigma $[12.0-16.0 \mathrm{~Hz}]$, beta $[16.0-25 \mathrm{~Hz}]$.

\section{Results}

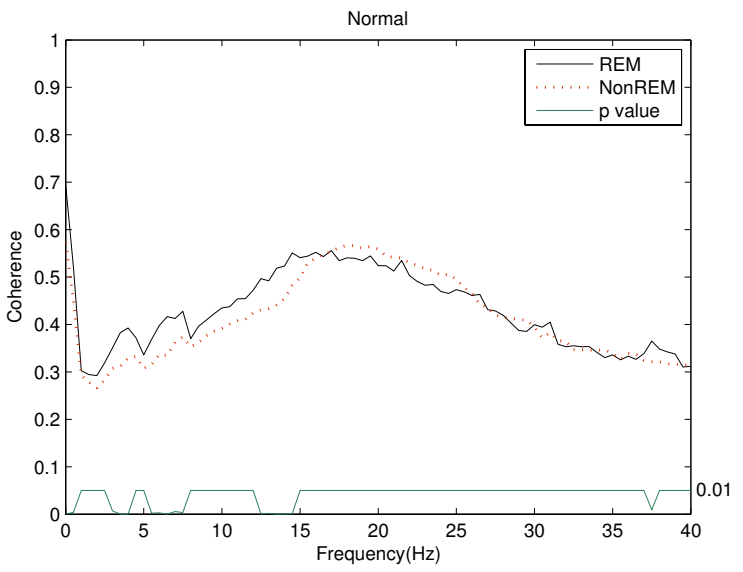

Figure 1. Mean values of Coherence $_{E C G-E E G}$ over the frequency range $(0-40 \mathrm{~Hz})$ for normal breathing events in NREM and REM sleep. $p$ values greater than 0.01 were not shown.

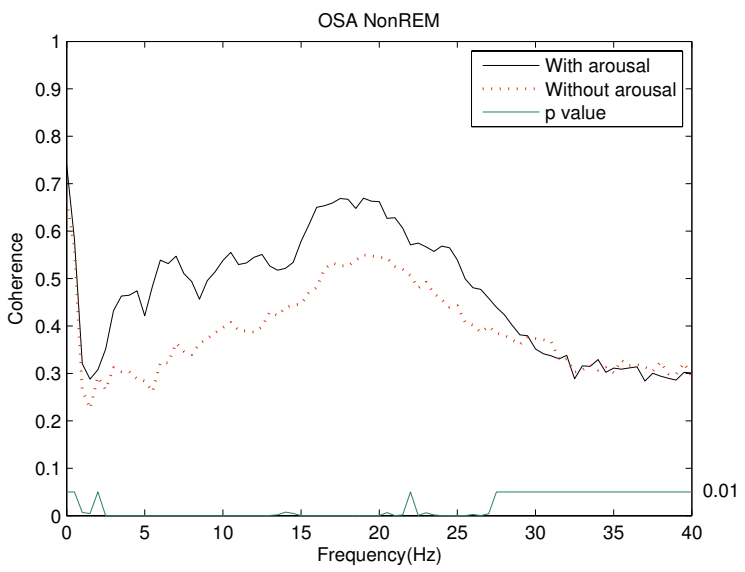

Figure 2. Mean values of Coherence ECG-EEG $_{\text {over the }}$ frequency range $(0-40 \mathrm{~Hz})$ for OSA events in NREM sleep with/without arousals. $p$ values greater than 0.01 were not shown. 


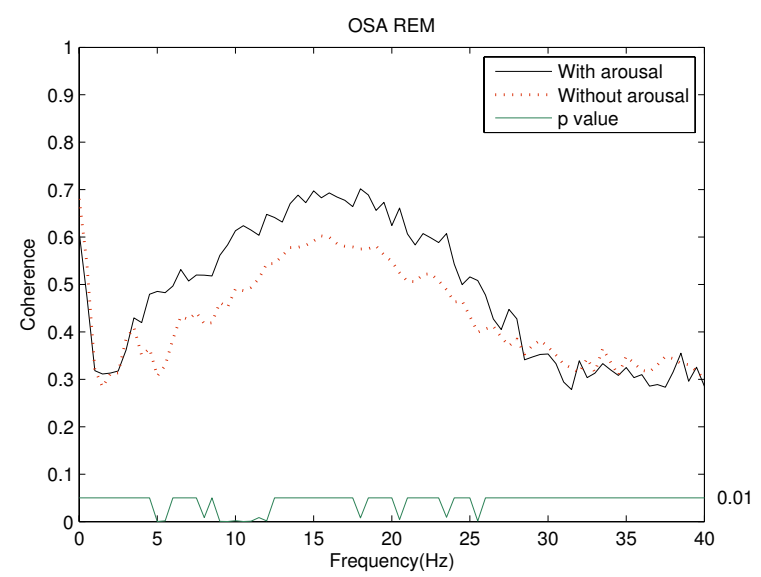

Figure 3. Mean values of Coherence $_{E C G-E E G}$ over the frequency range $(0-40 \mathrm{~Hz})$ for OSA events in NREM sleep with/without arousals. $p$ values greater than 0.01 were not shown.

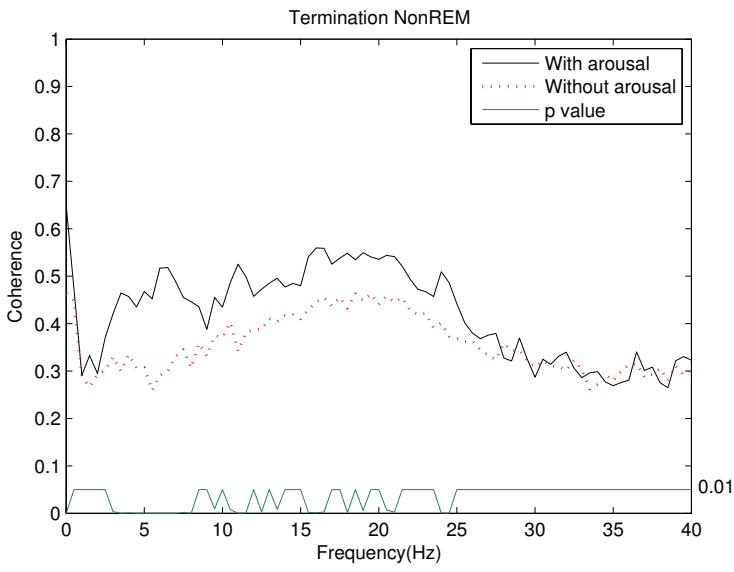

Figure 4. Mean values of Coherence ECG-EEG $_{\text {over the }}$ frequency range $(0-40 \mathrm{~Hz})$ for events following OSA termination in NREM sleep with/without arousals. $p$ values greater than 0.01 were not shown.

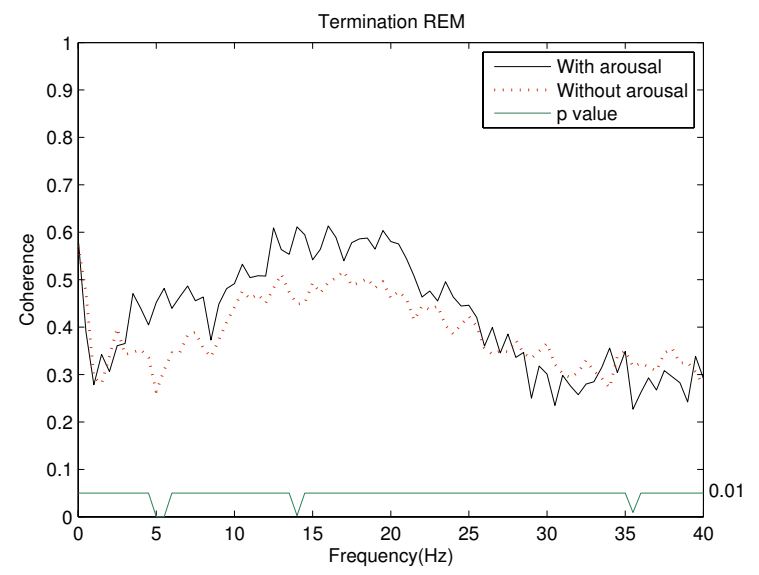

Figure 5. Mean values of Coherence $_{E C G-E E G}$ over the frequency range $(0-40 \mathrm{~Hz})$ for events following OSA termination in REM sleep with/without arousals. $p$ values are also shown for all frequencies. $p$ values greater than 0.01 were not shown.

Table 1. Mean and SD of Coherence ECG-EEG $_{\text {values over }}$ different frequency ranges during NREM and REM sleep for normal, OSA and the event following OSA termination. ANOVA test shows significance $(p<0.01)$ in all frequency bands over 5 groups in NREM and all bands except $0.5-3.0 \mathrm{~Hz}$ in REM. Posthoc test results shown as superscript meaning significant $(p<0.01)$ difference in that particular frequency band with the named group.

\begin{tabular}{|c|c|c|c|c|}
\hline Group & $\begin{array}{c}\text { Arousal } \\
\text { Status }\end{array}$ & $\begin{array}{l}\text { Freq } \\
(\mathrm{Hz})\end{array}$ & NREM & REM \\
\hline \multirow{5}{*}{$\mathrm{a}$} & \multirow{5}{*}{$\begin{array}{c}\text { Normal } \\
\text { Without } \\
\text { Arousal } \\
(\mathrm{NREM}=81 \\
\mathrm{REM}=108)\end{array}$} & $0.5-3.0$ & $0.35 \pm 0.07^{\text {bd }}$ & $0.40 \pm 0.09$ \\
\hline & & $3.0-8.0$ & $0.34 \pm 0.09^{\mathrm{bd}}$ & $0.39 \pm 0.09^{\text {bde }}$ \\
\hline & & $8.0-12.0$ & $0.39 \pm 0.12^{\text {bd }}$ & $0.44 \pm 0.12^{b}$ \\
\hline & & $12.0-16.0$ & $0.48 \pm 0.15^{\text {be }}$ & $0.53 \pm 0.14^{b}$ \\
\hline & & $16.0-25.0$ & $0.54 \pm 0.16^{\text {be }}$ & $0.51 \pm 0.14^{b}$ \\
\hline \multirow{5}{*}{ b } & \multirow{5}{*}{$\begin{array}{c}\text { OSA With } \\
\text { Arousal } \\
(\mathrm{NREM}=54 \\
\text { REM=24) }\end{array}$} & $0.5-3.0$ & $0.43 \pm 0.07^{\mathrm{ce}}$ & $0.39 \pm 0.08$ \\
\hline & & $3.0-8.0$ & $0.49 \pm 0.11^{\mathrm{ce}}$ & $0.49 \pm 0.13^{\mathrm{ce}}$ \\
\hline & & $8.0-12.0$ & $0.52 \pm 0.12^{\mathrm{ce}}$ & $0.60 \pm 0.09^{\text {cde }}$ \\
\hline & & $12.0-16.0$ & $0.56 \pm 0.14^{\mathrm{ce}}$ & $0.67 \pm 0.15^{\mathrm{e}}$ \\
\hline & & $16.0-25.0$ & $0.62 \pm 0.12^{\text {cde }}$ & $0.62 \pm 0.18^{\mathrm{e}}$ \\
\hline \multirow{5}{*}{$\mathrm{c}$} & \multirow{5}{*}{$\begin{array}{c}\text { OSA } \\
\text { Without } \\
\text { Arousal } \\
(\mathrm{NREM}=98 \\
\text { REM=59) }\end{array}$} & $0.5-3.0$ & $0.37 \pm 0.08$ & $0.41 \pm 0.08$ \\
\hline & & $3.0-8.0$ & $0.31 \pm 0.07^{\mathrm{d}}$ & $0.39 \pm 0.09$ \\
\hline & & $8.0-12.0$ & $0.39 \pm 0.11^{\mathrm{d}}$ & $0.48 \pm 0.10$ \\
\hline & & $12.0-16.0$ & $0.44 \pm 0.15$ & $0.58 \pm 0.13^{\mathrm{e}}$ \\
\hline & & $16.0-25.0$ & $0.51 \pm 0.14^{\mathrm{e}}$ & $0.53 \pm 0.12$ \\
\hline \multirow{5}{*}{ d } & \multirow{5}{*}{$\begin{array}{c}\text { Termin } \\
\text { With } \\
\text { Arousal } \\
(\mathrm{NREM}=54 \\
\text { REM=24) }\end{array}$} & $0.5-3.0$ & $0.40 \pm 0.09^{\mathrm{e}}$ & $0.38 \pm 0.09$ \\
\hline & & $3.0-8.0$ & $0.47 \pm 0.12^{\mathrm{e}}$ & $0.46 \pm 0.13$ \\
\hline & & $8.0-12.0$ & $0.46 \pm 0.11^{\mathrm{e}}$ & $0.48 \pm 0.13$ \\
\hline & & $12.0-16.0$ & $0.50 \pm 0.14^{\mathrm{e}}$ & $0.58 \pm 0.15$ \\
\hline & & $16.0-25.0$ & $0.51 \pm 0.14^{\mathrm{e}}$ & $0.53 \pm 0.16$ \\
\hline \multirow{5}{*}{$\mathrm{e}$} & \multirow{5}{*}{$\begin{array}{c}\text { Termin } \\
\text { Without } \\
\text { Arousal } \\
(\mathrm{NREM}=98 \\
\text { REM=59) }\end{array}$} & $0.5-3.0$ & $0.34 \pm 0.09$ & $0.39 \pm 0.09$ \\
\hline & & $3.0-8.0$ & $0.31 \pm 0.07$ & $0.34 \pm 0.09$ \\
\hline & & $8.0-12.0$ & $0.37 \pm 0.10$ & $0.43 \pm 0.11$ \\
\hline & & $12.0-16.0$ & $0.42 \pm 0.13$ & $0.48 \pm 0.15$ \\
\hline & & $16.0-25.0$ & $0.43 \pm 0.13$ & $0.46 \pm 0.14$ \\
\hline
\end{tabular}

\section{Discussion}

In summary, the major findings are as discussed below

1) Coherence $_{E C G-E E G}$ in normal breathing events are found to be significantly $(\mathrm{p}<0.01)$ different than that in OSA events with arousals in NREM sleep (over 0.5$25 \mathrm{~Hz}$ ) and in REM sleep (over 3.0-25 Hz). We can speculate that cardiovascular autonomic responses to OSA events may drive abnormal afferent feedback signals most likely interacting at the level of the brainstem. It has been reported [12] that fluctuations in delta power $(0.5-3.0 \mathrm{~Hz})$ which increases after the onset of NREM sleep and then decreases during REM sleep, are responsible for variability of arousal thresholds to airway 
occlusion.

2) Coherence $_{E C G-E E G}$ in OSA events with arousals are significantly different than that without arousals in NREM sleep over $0.5-25 \mathrm{~Hz}$ bands but in REM sleep over 3.0-12 Hz only. Sleep spindles with a frequency of 12-16 Hz occurs only during NREM sleep. Spindles are triggered by brainstem activity often in response to stimuli such as noise. They represent a sleep-stabilizing response to a potential arousal [10].

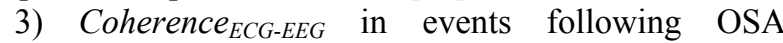
terminations with arousals are found to be significantly $(\mathrm{p}<0.01)$ different than that in events without arousals only in NREM sleep (over $0.5-25 \mathrm{~Hz}$ ). Sudden increase in EEG frequency is difficult to identify in REM sleep in which the transient reappearance of EMG activity indicates an arousal. Also, arousal threshold is higher in REM than in NREM sleep. We speculate that insignificant differences in REM are a result of cortical influences during phasic REM sleep that are related to increased brain activity during dreaming.

It is unclear what terminates OSAs which are not terminated by visible cortical arousals. Our results may speculate that autonomic arousals which are not represented at cortex, may actually be represented in a different way at the cortex but the changes cannot be detected with standard techniques. Also, association between arousal and upper airway opening in obstructive hypopneas-apneas, was found to be incidental [11].

The limitation of this study is that proper separation of the effect of cardiac electric field from heart cycle related brain potentials was not considered. Although the cardiac field artifact (CFA) in the EEG is not relevant in most clinical and experimental situations because of its small magnitude, but in connection with heart cycle-related EEG averaging it turns out to be a problem. In this study we have not used any computational method to remove the CFA from the averaged EEG data.

The results of this study will be useful in revealing the changes in the interconnection between the parameters of the analyzed signals that are related to sleep disordered breathing. For example, Cheyne-Stokes respiration is common in disorders of the cerebral hemisphere which reduces the inhibition of the medullary respiratory centres so that the reflex responses become accentuated. It could also be useful in detecting OSA events or OSA related arousals to characterize sleep fragmentation.

\section{Acknowledgements}

This study was supported by an Australian Research Council (ARC) Linkage Project with Compumedics Pty Ltd (LP0454378). The authors would like to thank all members of research and innovation team of Compumedics for providing sleep studies and their valuable advices, feedback and support.

\section{References}

[1] Jurysta F, van de Borne P, Migeotte PF, Dumont M, Lanquart JP, Degaute JP, and Linkowski P. A study of the dynamic interactions between sleep EEG and heart rate variability in healthy young men. Clin Neurophysiol 2003; 114: 2146-2155.

[2] Jurysta F, Lanquart JP, van de Borne P, Migeotte PF, Dumont M, Degaute JP and Linkowski P. The link between cardiac autonomic activity and sleep delta power is altered in men with sleep apnea-hypopnea syndrome. Am J Physiol Regulatory Integrative Comp Physiol 2006;291:1165-1171.

[3] Dingli K, Fietze I, Assimakopoulos T, Quispe-Bravo S, Witt C, Douglas NJ. Arousability in sleep apnoea/hypopnoea syndrome patients. Eur Respir J 2002; 20: 733-740.

[4] Roehr T, Spence DPS, Earis JE, Calverly PMA. Predictors of objective level of daytime sleepiness in patients with sleep-related breathing disorders. Chest 1989; 95:12021206.

[5] Dirlich G, Vogl L, Plaschke M, Strian F. Cardiac field effects on the EEG. Electroencephalogr Clin Neurophysiol 1997; 102:307-315.

[6] Dirlich G, Dietl T, Vogl L, Strian F. Topography and morphology of heart action-related EEG. Electroencephalography and Clinical Neurophysiology 1998;108(3): 299-305.

[7] AMERICAN ACADEMY OF SLEEP MEDICINE (AASM) TASK FORCE. Sleep-related breathing disorders in adults: recommendations for syndrome definition and measurement techniques in clinical research. Sleep 1999; 22:667-689.

[8] Rechtschaffen A and Kales A, eds. A Manual of Standardized Techniques and Scoring Systems for Sleep Stages of Human Subjects. National Institute of Health Washington: US govt printing press, 1968:204.

[9] American Sleep Disorders Association (ASDA) Task Force Report. EEG arousals: scoring rules and examples. Sleep 1992; 15:173-184.

[10] Gennaro LD, Ferrara M. Sleep spindles: an overview. Sleep Med Rev. 2003; 7(5): 423-40.

[11] Younes M. Role of Arousals in the Pathogenesis of Obstructive Sleep Apnea. Am J Respir Crit Care Med 2004; 169:623-633.

[12] Berry RB, Asyali MA, McNellis MI and Khoo MCK. Within-night variation in respiratory effort preceding apnea termination and EEG delta power in sleep apnea. J Appl Physiol 1998; 85:1434-1441.

Address for correspondence

Ahsan Khandoker

Dept. of Electrical \& Electronic Engg. The University of Melbourne, VIC -3010, Australia.

E-mail: a.khandoker@ee.unimelb.edu.edu.au 